\title{
The Effect of Probe Pressure on In Vivo Single Fiber Reflectance Spectroscopy
}

\author{
Sanaz Hariri Tabrizi ${ }^{{ }^{*}}$, Ali Akbar Shakibaei ${ }^{2}$ \\ ${ }^{1}$ Department of Medical Radiation Engineering, Shahid Beheshti University, Tehran, Iran \\ ${ }^{2}$ Department of Physics, Eastern Mediterranean University, Famagusta, Via Mersin10, Turkey
}

\author{
*Correspondence to \\ Sanaz Hariri Tabrizi, PhD; \\ Department of Medical Radiation \\ Engineering, Shahid Beheshti \\ University, Evin, Tehran, Iran \\ Tel: +98-2129904220; \\ Email: S_HaririTabrizi@sbu.ac.ir
}

Published online 27 October 2016

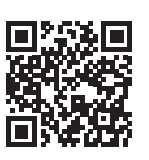

\begin{abstract}
Introduction: Single fiber reflectance spectroscopy (SFRS) is a noninvasive procedure to quantitate tissue absorption and scattering properties. It can be used to diagnose different diseases such as malignancy and pre-cancerous conditions. The measurement is done with a fiber optic probe in contact with the tissue surface. Herein, the effect of probe pressure on the extracted parameters from human lip spectra was studied.

Methods: Thirty-three normal subjects were examined with three exerted pressure levels on the right, middle and left parts of their lips.

Results: The results showed variation of spectroscopic parameters with different pressure levels. However, the effect was seen between a very mild contact (pressure 1) and the other reasonably practical pressure levels normally used in the medical centers.

Conclusion: SFRS can be used as a reliable diagnostic tool in clinics.

Keywords: Spectroscopy; Reflectance; Probe pressure; Lip measurement.
\end{abstract}

\section{Introduction}

Reflectance spectroscopy is a noninvasive method to determine the scattering and absorption properties of a turbid medium, such as tissue. This information can be used to describe aspects of the tissue physiology and morphology. ${ }^{1}$ Single fiber reflectance spectroscopy (SFRS) uses a single fiber for the delivery and detection of light. The advantages of SFRS over other techniques such as spatially resolved diffuse reflectance spectroscopy (DRS) ${ }^{2}$ are the simplicity of the setup and the small dimensions of the fiber-optic probes. $^{3}$ It has shown its potential in diagnosis of cancer and pre-cancer in lung lymph nodes, ${ }^{4}$ brain, ${ }^{5}$ skin $^{6}$ and uterine cervix. ${ }^{7}$

In order to obtain clinically useful information about the measured tissue, the spectroscopy results must depend solely on the tissue characteristics rather than environmental parameters, such as probe pressure. ${ }^{8}$ Probe pressure effect on fluorescence $e^{9-12}$ and reflectance $e^{9,11,13,14}$ spectroscopy has been studied by several authors. Nath et $\mathrm{al}^{12}$ and Rivoire et $\mathrm{al}^{10}$ investigated this effect on fluorescence spectra acquired from the human cervix. By application of three pressure levels they did not find significant difference in the fluorescence intensity or line shape acquired from the patients.

Reif et $\mathrm{al}^{13}$ investigated the effect of five pressure levels on the right and left thigh muscles of ten mice. The probe came in contact with the muscle after removal of the skin.
They found that the absorption and scattering properties of the mouse thigh muscle depended on the probe pressure.

Ti et $\mathrm{al}^{11}$ tested both fluorescence and reflectance spectroscopy dependence on the probe pressure using six animal tests. The heart and liver of the anesthetized rats were exposed to 5 probe pressure levels during surgery. Their results showed a significant spectral alteration of diffuse reflectance and fluorescence spectra after exceeding a threshold pressure. They concluded that such changes may be highly tissue-dependent and the potential effect should be considered during in vivo studies.

Delgado Atencio et $\mathrm{al}^{14}$ and Lim et $\mathrm{al}^{9}$ investigated the probe pressure effect on human skin DRS spectra. Delgado Atencio et al ${ }^{14}$ collected the spectra from 45 normal forearm skins and one skin lesion. They compared the effect of pressure on the spectra in 6 different pressure levels. Their results showed a decrease in intensity of diffuse reflectance above $600 \mathrm{~nm}$ in $83 \%$ of cases while no remarkable difference was observed in spectral band of 400-600 nm.

Lim et $\mathrm{al}^{9}$ studied the short-term $(<2$ seconds) and longterm ( $>30$ seconds) effects of six probe pressure levels on DRS and fluorescence measurements. The studied samples included forehead, neck and finger skin of five normal volunteers. They found a site-specific effect up to $10 \%$ but negligible short-term effect of light probe pressure. 
This study was done to investigate the effect of probe pressure on the SFRS spectra. SFRS has a shorter pathlength than DRS which has been studied by other researchers. The studied tissue was the human lip to simulate the effect of pressure on uterine cervix tissue with comparative characteristics of epithelium layer. Herein, the effects of 3 different probe pressure levels during the lower lip measurements of normal volunteers were evaluated.

\section{Methods}

Experimental Setup

The SFRS system setup has been described previously. ${ }^{7}$ Briefly, it included an optical fiber spectrophotometer and a tungsten halogen light source. A pair of bifurcation connected the two devices to a common fiber by two $200 \mu \mathrm{m}$ diameter optical fibers. The common $1000 \mu \mathrm{m}$ diameter contact probe was enclosed in a stainless steel tube with a $1.6 \mathrm{~mm}$ outer diameter and the tip was polished at $15^{\circ}$ angle to reduce the specular reflectance (Figure 1).

At the distal end of the probe a spring and a holder were designed in order to apply a specific pressure to the tissue. Before the experiment, the linearity of the spring was verified. The measurements were calibrated using a high scattering solid phantom (Titanium dioxide $\left[\mathrm{TiO}_{2}\right]$ ) and water within a dark container. The calibrated signal was quotient of the measured spectra to the phantom measurement while the water measurement was subtracted from both. ${ }^{16}$ Spectra were collected and saved using an interface written by LabView code (version 7.1; National Instruments).

\section{In Vivo Measurements}

Thirty-three normal volunteers ( 8 females, 25 males) with $30 \pm 8$ years old age were included in this study. Three different pressure levels were applied to the right, middle and left parts of the lower lip of the subjects. Measurements were acquired by the same user to eliminate inter-user variability. A colorful indicator was positioned under the spring of the probe holder to determine the applied pressure level. The highest pressure (3) was chosen as not to hurt the subject while the lowest one (1) was

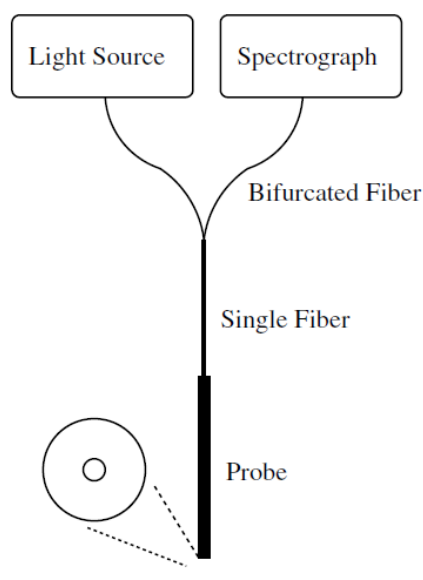

Figure 1. Schematic of the Single Fiber Reflectance Spectroscopy (SFRS) System Setup. ${ }^{15}$ such that a very mild contact was established between the probe tip and tissue. The middle pressure (2) was chosen between these two extremes. Five SFRS spectra were averaged at each site to reduce the noise.

\section{Data Analysis}

A semi-empirical model for SFRS spectra analysis has been described previously ${ }^{16}$ and it was applied successfully in cervical tissue. ${ }^{7}$ Because of the similarity of the lower lip tissue with the cervical tissue, the same model was used to analyze the measured spectra. However, melanin was added to the model in addition to previously described absorbers, including blood and beta-carotene. The fitting parameters included Mie scattering amplitude (Mie-amp), Mie scattering slope (Mie-sl), Rayleigh scattering amplitude (Ray-amp), blood volume fraction (Bl-vol), average vessel diameter (Ves-diam), melanin concentration (Mel), beta-carotene concentration (B-car) and blood oxygen saturation $\left(\mathrm{StO}_{2}\right)$. Parameter estimation was done using a Levenberg-Marquardt (LM) algorithm that was scripted into LabView code. Figure 2 shows a typical lip spectrum as well as its fitting curve.

A total of 288 spectra were obtained from 33 subjects. Among these spectra the ones with bad fitting (mean squared error $>100$ ) were excluded. Also, the parameters with high uncertainty and low level of signal to noise were discarded. The criterion for exclusion of each parameter depended on the frequent error associated with it among the measurements. The effect of contact probe pressure on the fitting parameters was evaluated by calculating the significance of the difference between fitting parameter mean values in each pressure group. It was done using Kruskal-Wallis (KW) one-way analysis of variance test. The statistical significance was set to $P<0.05$. Figure 3

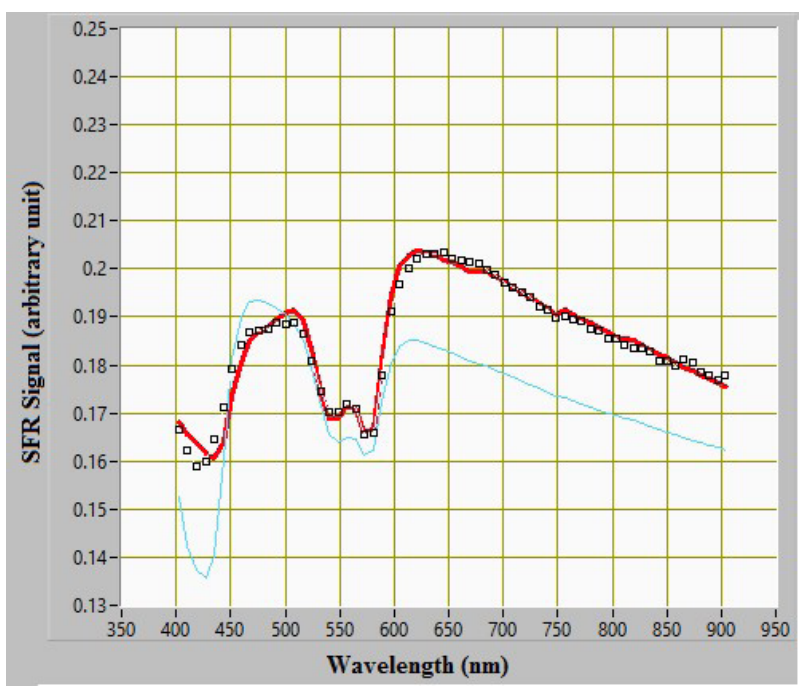

Figure 2. A Typical Middle Part of the Lip Spectrum Obtained With the Second Pressure Level Shown in LabView Interface. Black squares indicate the data points of SFRS spectrum as a function of wavelength. The blue and red lines are the fitting curves obtained by the initial guess fitting parameters and after application of Levenberg-Marquardt (LM) algorithm, respectively. 


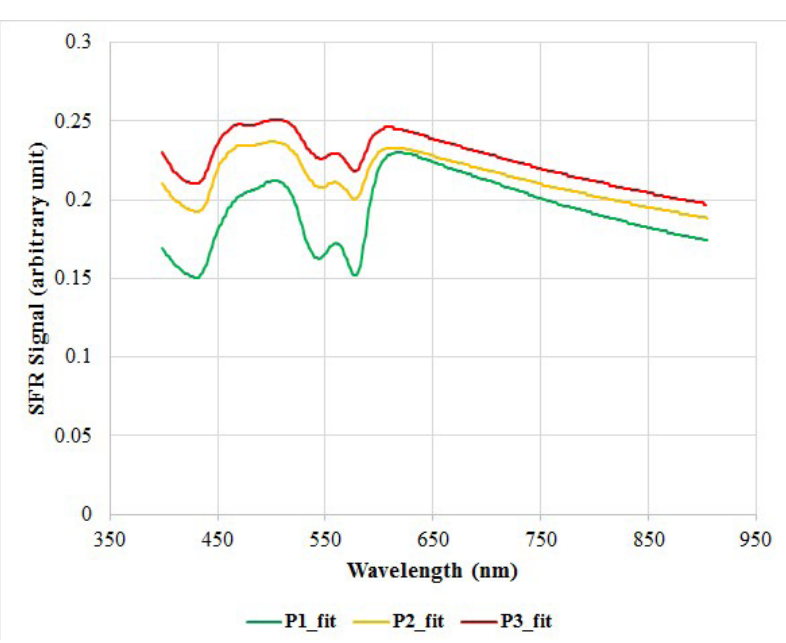

Figure 3. Comparison Between Spectra Acquired With the Three Pressure Levels Applied to the Middle Part of the Lip of the Volunteer Shown in Figure 2.

shows the fitted spectra of the middle part of the lip measurement of a male volunteer for the three applied pressure levels.

\section{Results}

The difference of fitting parameters between the three pressure levels was analyzed for each measurement site. The number of acceptable data points for different pressure levels is summarized in Table 1. Exclusion of unacceptable values resulted in different number of analyzed parameters for each pressure category. Among the three measurement sites, Table 2 presents the results for middle part of the lip measurements. The mean and standard deviation of the fitting parameters for each pressure level as

Table 1. The Number of Acceptable Data Points for Statistical Analysis of Each Site and Pressure Level

\begin{tabular}{lcccc}
\hline $\begin{array}{l}\text { Measurement Site on the } \\
\text { Lip }\end{array}$ & \multicolumn{3}{c}{ Pressure Level } & \multirow{2}{*}{ Total } \\
\cline { 2 - 4 } & $\mathbf{1}$ & $\mathbf{2}$ & $\mathbf{3}$ & \\
\hline Left & 31 & 32 & 30 & 93 \\
Middle & 32 & 30 & 30 & 92 \\
Right & 30 & 31 & 26 & 87 \\
\hline
\end{tabular}

well as their corresponding KW $P$ values are shown.

In a one-way analysis of variance, the comparison is made between the means of several groups to test the hypothesis that they are all the same. But sometimes, the information about the significance of difference between pairs of mean values is of concern. Therefore, a multiple comparison procedure can be performed. Table 3 shows the significantly different pairs for each parameter among the three pressure levels for the right, middle and left parts of the lip measurements. In other words, the check marks in the table show the pressure pairs which $95 \%$ CI of difference of their mean values did not include zero. The data was analyzed using MATLAB software.

\section{Discussion}

Table 2 shows that all the spectroscopic parameters except Ray-amp were affected by different pressure levels of the contact probe during the middle part of the lip measurement. Increase in probe pressure resulted in increase of scattering amplitude in contrast with all other parameters. Reif et al found that the blood vessel radius, oxygen saturation and Mie slope decreased while the reduced scattering coefficient at $700 \mathrm{~nm}$ increased with increasing the probe pressure. ${ }^{13}$ Our results are in agreement with them. Decrease in absorption and reduced scattering coefficients with increasing the pressure is consistent with the results obtained by Lim et al. ${ }^{9}$ However, the trend of scattering was reversed after $500 \mathrm{~nm}$ in their study.

The comparison was made in detail in Table 3. It is shown that the significant difference is only between the first pressure level and the others or, only for one case (Mieamp during middle part of the lip measurement), between the first and third pressure levels. This effect can be seen in Figure 3 in which the difference between P1 and the others is more pronounced than between P2 and P3. It means that the mostly used pressures during a clinical experiment (pressures 2 and 3 ) do not produce any difference in the extracted parameters from the measured SFRS spectra. It is a good piece of news because a very mild contact with the tissue, as applied in the pressure level 1 , is not a common practice for clinical use.

Less effect of probe pressure on spectroscopic parameters of SFRS compared with DRS can be attributed to the

Table 2. The Mean and Standard Deviation of the Spectroscopic Parameters for Middle Part of the Lip Measurements and Their Corresponding KW Test $P$ Values

\begin{tabular}{lcccc}
\hline \multirow{2}{*}{ Parameters } & \multicolumn{3}{c}{ Mean \pm Standard Deviation for Pressures } & \multirow{2}{*}{$\boldsymbol{P}$ Value } \\
\cline { 2 - 4 } Mie-amp (-) & $\mathbf{1}$ & $\mathbf{2}$ & $\mathbf{3}$ & 0.046 \\
Mie-sl (-) & $0.474 \pm 0.089$ & $0.513 \pm 0.069$ & $0.527 \pm 0.064$ & 0.000 \\
Ray-amp (-) & $-1.212 \pm 0.281$ & $-0.934 \pm 0.184$ & $-0.863 \pm 0.144$ & 0.113 \\
Bl-vol (-) & $0.004 \pm 0.009$ & $0.005 \pm 0.008$ & $0.005 \pm 0.008$ & 0.000 \\
Ves-diam (mm) & $0.017 \pm 0.006$ & $0.008 \pm 0.005$ & $0.008 \pm 0.004$ & 0.000 \\
Mel $(\mathrm{mM} . \mathrm{cm})$ & $0.021 \pm 0.006$ & $0.015 \pm 0.006$ & $0.015 \pm 0.005$ & 0.029 \\
B-Car $(\mu \mathrm{M})$ & $0.167 \pm 0.147$ & $0.097 \pm 0.081$ & $0.084 \pm 0.054$ & 0.000 \\
$\mathrm{StO}_{2}(\%)$ & $12.03 \pm 5.25$ & $6.89 \pm 3.31$ & $7.94 \pm 3.84$ & 0.003 \\
\hline
\end{tabular}


Table 3. The Significantly Different Average Parameters for Left, Middle and Right Part of the Lip Measurements

\begin{tabular}{|c|c|c|c|c|c|c|c|c|c|c|}
\hline \multirow{3}{*}{ Parameters } & \multirow[b]{3}{*}{ Pressure Levels } & \multicolumn{9}{|c|}{ Measurement Site } \\
\hline & & \multicolumn{3}{|c|}{ Left } & \multicolumn{3}{|c|}{ Middle } & \multicolumn{3}{|c|}{ Right } \\
\hline & & 1 & 2 & 3 & 1 & 2 & 3 & 1 & 2 & 3 \\
\hline \multirow{3}{*}{ Mie-amp } & 1 & & & & & & $\checkmark$ & & & \\
\hline & 2 & & & & & & & & & \\
\hline & 3 & & & & $\checkmark$ & & & & & \\
\hline \multirow{3}{*}{ Mie-sl } & 1 & & $\checkmark$ & $\checkmark$ & & $\checkmark$ & $\checkmark$ & & $\checkmark$ & $\checkmark$ \\
\hline & 2 & $\checkmark$ & & & $\checkmark$ & & & $\checkmark$ & & \\
\hline & 3 & $\checkmark$ & & & $\checkmark$ & & & $\checkmark$ & & \\
\hline \multirow{3}{*}{ Ray-amp } & 1 & & & & & & & & & \\
\hline & 2 & & & & & & & & & \\
\hline & 3 & & & & & & & & & \\
\hline \multirow{3}{*}{ Bl-vol } & 1 & & $\checkmark$ & $\checkmark$ & & $\checkmark$ & $\checkmark$ & & $\checkmark$ & $\checkmark$ \\
\hline & 2 & $\checkmark$ & & & $\checkmark$ & & & $\checkmark$ & & \\
\hline & 3 & $\checkmark$ & & & $\checkmark$ & & & $\checkmark$ & & \\
\hline \multirow{3}{*}{ Ves-diam } & 1 & & $\checkmark$ & $\checkmark$ & & $\checkmark$ & $\checkmark$ & & $\checkmark$ & $\checkmark$ \\
\hline & 2 & $\checkmark$ & & & $\checkmark$ & & & $\checkmark$ & & \\
\hline & 3 & $\checkmark$ & & & $\checkmark$ & & & $\checkmark$ & & \\
\hline \multirow{3}{*}{ Mel } & 1 & & $\checkmark$ & $\checkmark$ & & & & & $\checkmark$ & $\checkmark$ \\
\hline & 2 & $\checkmark$ & & & & & & $\checkmark$ & & \\
\hline & 3 & $\checkmark$ & & & & & & $\checkmark$ & & \\
\hline \multirow{3}{*}{ B-Car) } & 1 & & $\checkmark$ & $\checkmark$ & & $\checkmark$ & $\checkmark$ & & & \\
\hline & 2 & $\checkmark$ & & & $\checkmark$ & & & & & \\
\hline & 3 & $\checkmark$ & & & $\checkmark$ & & & & & \\
\hline \multirow{3}{*}{$\mathrm{StO}_{2}$} & 1 & & & & & $\checkmark$ & $\checkmark$ & & $\checkmark$ & $\checkmark$ \\
\hline & 2 & & & & $\checkmark$ & & & $\checkmark$ & & \\
\hline & 3 & & & & $\checkmark$ & & & $\checkmark$ & & \\
\hline
\end{tabular}

smaller sampling volume. ${ }^{9}$ While the number of illumination and collection optical fibers ranges from two ${ }^{13}$ to sev$\mathrm{en}^{14}$ in other similar studies, SFRS uses a single fiber for both illumination and collection of the spectra. The other reason is the shorter time during which the pressure was applied. While the pressure application duration differs from 5 seconds to 2 minutes in other studies, ${ }^{9,11,13,14}$ the acquisition and pressure application time was about one second in this study. It is long enough to collect 5 spectra from the tissue and short enough not to induce a significant effect on the collected data. It is consistent with the negligible short-term effect of probe pressure on tissue characteristics. ${ }^{9}$

In order to reduce the tissue-specific variations, ${ }^{9,11}$ the lip tissue was chosen for the experiment. It has comparable characteristics to uterine cervix which is under investigation by the authors. ${ }^{7,8}$ Also, the tissue was tested in its normal situation rather than exposing to the probe barely like other studies. ${ }^{11,13}$ Investigation of the sole effect of probe pressure on a comparable tissue and with similar experimental setup, confirmed the previous cervical tissue results. No significant difference was seen between different probe pressures exerted by ten clinicians in diagnosis of cervical pre-cancer using SFRS. ${ }^{8}$

\section{Conclusion}

The influence of probe pressure on the single fiber reflectance spectra has been studied. SFRS is a simple and cost-effective method for diagnosis of malignancies and pre-malignancies. While the probe pressure influences most of the SFRS spectroscopic parameters, the difference is seen between the lowest pressure level and the more reasonable ones. It shows the potential of SFRS system for diagnostic purposes in clinics without being concerned about the interfering factor of pressure variation.

\section{Ethical Considerations}

None.

\section{Conflict of Interests}

The authors report no conflict of interest.

\section{Acknowledgments}

We would like to thank all the participants in this study. Also, we would like to thank Shahid Beheshti University staff and faculty for providing required facilities for the study, especially Dr. SMR Aghamiri.

\section{References}

1. Amelink A, Kaspers OP, Sterenborg HJ, van der Wal JE, Roodenburg JL, Witjes MJ. Non-invasive measurement of the morphology and physiology of oral mucosa by use of optical spectroscopy. Oral Oncol. 2008;44(1):65-71.

2. Doornbos RM, Lang R, Aalders MC, Cross FW, Sterenborg HJ. The determination of in vivo human tissue optical properties and absolute chromophore concentrations using spatially resolved steady-state diffuse reflectance spectroscopy. Phys Med Biol. 1999;44(4):967-981.

3. Canpolat M, Mourant JR. Particle size analysis of turbid 
media with a single optical fiber in contact with the medium to deliver and detect white light. Appl Opt. 2001;40(22):3792-3799.

4. Kanick SC, van der Leest C, Djamin RS, et al. Characterization of mediastinal lymph node physiology in vivo by optical spectroscopy during endoscopic ultrasound-guided fine needle aspiration. J Thorac Oncol. 2010;5(7):981-987.

5. Canpolat M, Akyüz M, Gökhan GA, Gürer EI, Tuncer R. Intra-operative brain tumor detection using elastic light single-scattering spectroscopy:a feasibility study. J Biomed Opt. 2009;14(5):054021.

6. Canpolat M, Akman-Karakaş A, Gökhan-Ocak GA, Başsorgun IC, Akif Çiftçioğlu M, Alpsoy E. Diagnosis and demarcation of skin malignancy using elastic light singlescattering spectroscopy: a pilot study. Dermatol Surg. 2012;38(2):215-223.

7. Hariri Tabrizi S, Mahmoud Reza Aghamiri S, Farzaneh F, Amelink A, Sterenborg HJ. Single fiber reflectance spectroscopy on cervical premalignancies: the potential for reduction of the number of unnecessary biopsies. J Biomed Opt. 2013;18(1):017002.

8. Hariri Tabrizi S, Farzaneh F, Aghamiri SMR. Applicability of optical reflectance spectroscopy for detection of precancerous lesions in uterine cervix in vivo. Arch Iran Med. 2014;17(9):602-607.

9. Lim L, Nichols B, Rajaram N, Tunnell JW. Probe pressure effects on human skin diffuse reflectance and fluorescence spectroscopy measurements. J Biomed Opt.
2011;16(1):011012. doi:10.1117/1.3525288.

10. Rivoire K, Nath A, Cox D, Atkinson EN, Richards-Kortum $\mathrm{R}$, Follen $\mathrm{M}$. The effects of repeated spectroscopic pressure measurements on fluorescence intensity in the cervix. Am J Obstet Gynecol. 2004;191:1606-1617.

11. Ti Y, Lin WC. Effects of probe contact pressure on in vivo optical spectroscopy. Opt Exp. 2008;16:4250-4262.

12. Nath A, Rivoire K, Chang S, et al. Effect of probe pressure on cervical fluorescence spectroscopy measurements. J Biomed Opt. 2004;9:523-533.

13. Reif R, Amorosino MS, Calabro KW, A'Amar O, Singh SK, Bigio IJ. Analysis of changes in reflectance measurements on biological tissues subjected to different probe pressures. J Biomed Opt. 2008;13:010502.

14. Delgado Atencio JA, Orozco Guillén EE, Vázquez y Montiel S, et al. Influence of probe pressure on human skin diffuse reflectance spectroscopy measurements. Opt Mem Neural Networks. 2009;18(1):6-14. doi:10.3103/ S1060992X09010020.

15. Kanick SC, Sterenborg HJ, Amelink A. Empirical model of the photon path length for a single fiber reflectance spectroscopy device. Opt Exp. 2009;17(2):860-871.

16. Kanick SC, Gamm UA, Schouten M, Sterenborg HJ, Robinson DJ, Amelink A. Measurement of the reduced scattering coefficient of turbid media using single fiber reflectance spectroscopy:fiber diameter and phase function dependence. Biomedical Optics Express. 2011;2(6):16871702. doi:10.1364/BOE.2.001687. 